\title{
DETERMINING TASK OPTIMAL MODULAR ROBOT ASSEMBLY CONFIGURATIONS
}

\author{
I-Ming Chen \\ School of Mechanical and Production Engineering \\ Nanyang Technological University \\ Singapore 2263 \\ Republic of Singapore
}

Joel W. Burdick

Division of Engineering and Applied Science

MS 104-44 Caltech

Pasadena, CA 91125

U. S. A.

\begin{abstract}
A "modular" robotic system consists of standardized joint and link units that can be assembled into a number of different kinematic configurations. Given a predetermined set of modules, this paper considers the problem of finding an "optimal" module assembly configuration for a specific task. We formulate the solution as a discrete optimization procedure. The formulation is based on an assembly incidence matrix representation of a modular robot and a general task-oriented objective function that can incorporate many realistic task criteria. Genetic algorithms (GA) are employed to solve this optimization problem, and a canonical method to represent a modular assembly in terms of genetic strings is introduced. An example involving a 3DOF manipulator configuration is presented to demonstrate the feasibility of this approach.
\end{abstract}

\section{Introduction}

A modular reconfigurable robotic system is a collection of various sub-assemblies, at the level of links and joints, that can be easily separated and reassembled into different configurations through standardized connecting interfaces. By reconfiguring the modules, different robots can be created so as to suit a diversity of task requirements. Several prototype modular robotic systems have been built and demonstrated $[3,5,12,14]$.

Let the arrangement of modules in a modular robot be called an assembly configuration. We consider the taskoriented optimal configuration problem in this paperi.e., how to to find an optimal assembly configuration from a set of modules for a specific task. We assume that the set of modules is fixed at plamning time, and thus only freedom available for optimization is the recombination and rearrangement of modules. For simply designed module sets, the total number of possible assembly configurations can be manually enumerated, and this set of robot configurations can be easily tested against the task requirements. As the module design becomes more complicated and versatile, the possible number of the robot's assembly configurations grows tremendously. An efficient and systematic method to find an optimal configuration is thus necessary.

Here we introduce a general framework for solving the optimal assembly configuration problem. We define a generally applicable task related objective function which evaluates a modular robot assembly configuration for a given task while avoiding sub-assemblies with undesirable kinematic properties. This function could be applied to all of the unique assembly configurations which result from the algorithm outlined in $[1,2]$. Alternatively, we can use this function as the basis for discrete or combinatorial optimization method. In this

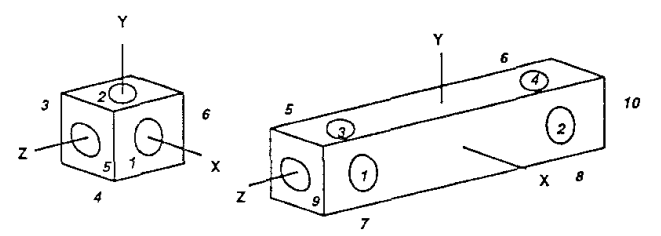

Figure 1: Link modules-a cubic box and a prism

paper, we employ genetic algorithms because of the discrete nature of the assembly configuration set.

Paredis and Khosla [11] have considered task-based robot design for fixed configuration robots. In their formulation, all design parameters are assumed continuous values, and hence conventional optimization techniques can be employed. In the problem posed here, the search space, the set of assembly configurations, is a discrete set.

\section{Conceptual Module Models}

A conceptual module model set based on the features found in many real implementations is introduced $[1,2]$. The module set consists of link and joint modules only. The following 1 - or 2-DOF joint modules are considered: revolute joint $(R)$, prismatic joint $(P)$, helical joint $(\mathrm{H})$, and cylindrical joint $(\mathrm{C})$. Joint modules are connected to the link modules through connecting ports and link modules possess multiple joint connections. We further assume that link modules have symmetrical geometry and symmetrically located connecting ports (though modules with only two connecting ports and no symmetry can be handles as well). The connecting ports are labeled accordingly. The symmetry design allows link modules to be re-oriented without altering the robot kinematics. For illustration purposes, we assume only two types of link modules are available: square prisms (10 ports) and cubic box units ( 6 ports), which are shown in Fig. 1. Other objects can be similarly treated.

\section{Modular Robot Assembly Representation}

To describe the location of a joint on a multi-port link module, we use a function termed assembly pattern. Let PORT be the set of port numbers on a link and ATT be the set of connecting status on a port. ATT contains eligible joint types and a zero indicating an empty port. For our modular robotic system, $\mathrm{ATT}=\{0, R, H, C, P\}$. For a cubic box, PORT $=\{1,2,3,4,5,6\}$.

Definition 1 The assembly pattern on a link module is an injection mapping

$$
f: \text { PORT } \rightarrow \text { ATT. }
$$




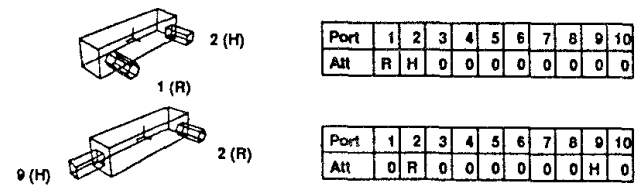

Figure 2: Two assembly pattern examples

If the port is connected, $f$ will assign a joint type. If it is empty, a zero will be assigned. Because of the link symmetry, many assembly patterns can be reoriented in a way that they function identically in a large robot structure. An equivalence based on the symmetric rotations of link modules was defined on $f$ in [1] to classify distinct link-joint assembly patterns. Chen [2] introduced an enumeration algorithm, OrbitEnumerate, based on this equivalence, to list all distinct assembly patterns on a link module with a prescribed number and types of joints. Examples of assembly patterns are shown in Fig. 2.

The connectivity of individual links and joints, or the topology, in a robot mechanism can be represented by a kinematic graph, in which vertices are links and edges are joints [4]. This graph, in turn, can be expressed as a vertex-edge incidence matrix [16] which contains only 0 s and $1 \mathrm{~s}$. The number of columns and rows in this matrix are equal to the number of edges and vertices in the graph respectively. Entry $(i, j)$ is equal to 1 if edge $j$ is incident on vertex $i$, or it is equal to zero otherwise.

Definition 2 Let $G$ be the graph of a modular robot and let $M(G)$ be its incidence matrix. The assembly configuration of this robot can be represented by an assembly incidence matrix, $A(G)$, obtained by replacing every entry of 1 in $M(G)$ with a non-zero integer, $k \in$ PORT. The zero entries remain unchanged. $a_{i j}=k$ indicates that joint $e_{j}$ is attached to port $k$ of link $v_{i}$; $a_{i j}=0$, otherwise.

For heterogeneous modular robots, which contain different kinds of link and joint modules, the assembly incidence matrix is augmented with an additional row and column that specifies the type of link and joint modules and is called the extended assembly incidence matrix (eAIM) [2]. Examples of assembly configurations of the following AIMs are shown in Fig. 3.

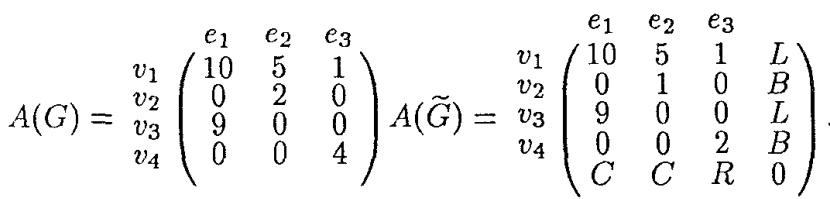

As pointed out in [1], different AIMs may have identical kinematic properties, such as the workspace and location of joint singularities. These AIMs are classified by an equivalence relation in order to reduce the number of unique robot assembly configurations. Two AIMs are said to be equivalent if the underlying graphs are isomorphic and the corresponding link assembly patterns are equivalent. The configuration enumeration algorithm, RobotEnumerate, stated in [2] utilized this equivalence definition. The output is a set of kinematically unique robot assembly configurations.
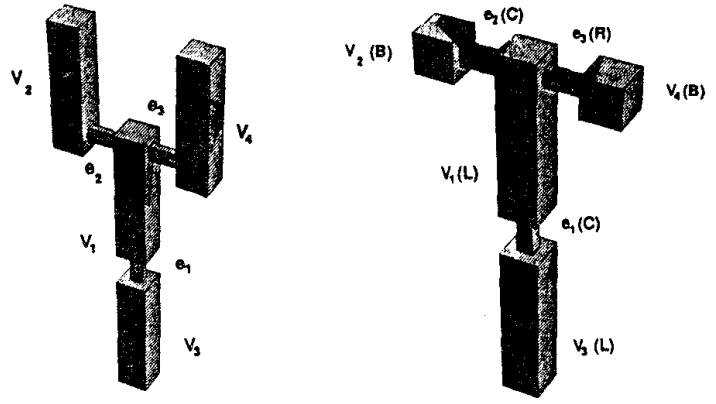

Figure 3: Different assembly configurations

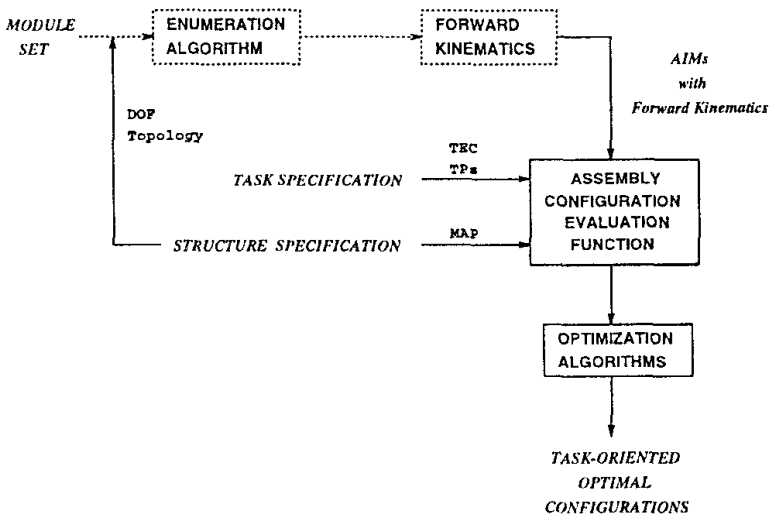

Figure 4: The problem solving scheme

\section{General Formulation}

To find an optimal solution, we wish to formulate an objective function that will evaluate how well an assembly configuration can accomplish an assigned task. Ideally, the form of the evaluation function should be general enough so that it is applicable to a wide variety of tasks, but flexible enough to incorporate different criteria which may be task specific. We term this function an assembly configuration evaluation function (ACEF). In effect, the ACEF is used to evaluate every unique assembly configuration generated by RobotEnumerate for a certain task requirement. The assembly configuration with the greatest ACEF value is deemed optimal. Then, this function can be used as the basis for a discrete optimization scheme. The remainder of this paper focuses primarily on the task evaluation function and its use for a discrete optimization procedure.

It is also important to note that from a given set of modules it may be possible to construct robots with various topologies-e.g., robots with serial or parallel kinematic structures. Even with a fixed robot topology class, the number of degrees of freedom (DOF) can alter the kinematic functionality of the system. For simplicity, we discuss the case of fixed topology and fixed number of DOF. Fig. 4 depicts the scheme to solve the task-oriented optimal configuration problem for the case of a specific robot topology. The desired robot topology and the number of DOFs are fed into the configuration enumeration algorithm in advance. A quantifiable task definition and task evaluation criteria (TEC) are parameters to the ACEF. Furthermore, an 
auxiliary function, termed the module assembly preference (MAP), is defined on individual assembly patterns to filter out those with inappropriate or undesirable kinematic features. The overall optimization problem becomes:

GIVEN: a robot task, a TEC, a MAP, and prescribed robot topology,

FIND: an AIM in the assembly configuration set whose $A C E F$ value achieves a maximum

If a set of robot configurations is given in advance, the enumeration algorithm can be bypassed, and the optimization procedure employed. The following sections explain the details of choosing task definitions, TEC, and MAPs. We use a serial type fixed base modular arm with revolute joints to demonstrate this problem solving strategy.

\section{Task Specifications}

\section{DEFINITION OF ROBOT TASKS}

A robot task is defined as a collection of working points, $\mathrm{w}_{p}$, in the operation space $R^{3}$ [8] or a collection of the end-effector positions/orientations $\mathrm{w}_{p}=$ $(x, y, z, \theta, \phi, \psi)$, where $\theta, \phi$, and $\psi$ are Euler angles representing the orientation of the end-effector frame. If the robot is to follow a trajectory, this task can be approximated by a set of points along the path.

\section{TAsk Evaluation CRITERIA (TEC)}

Many monotonically increasing local kinematic performance measures can be employed for task evaluation $[9,10,15]$. These measures are typically evaluated at a particular working point with a specified mechanism posture. Many measures are based on the mechanism's Jacobian matrix, J-e.g., the manipulability measure, $\operatorname{det}\left(J J^{T}\right)^{1 / 2}[15]$ and the condition number or minimum singular value of $J[9,10]$. Other configuration dependent criteria are possible.

Let $\mu_{i}$ be the value of a modular robot performance measure at task point $i$. For a collection of $n$ task points, we choose to define the total task performance, $\mu$, to be the smallest $\mu_{i}$ among the task points, i.e,

$$
\mu=\min _{i \in\{1, \cdots, n\}} \mu_{i}
$$

$\mu$ represents the worst case among the collection of task points. Since $\mu_{i} \geq 0$, by definition, $\mu \geq 0$. This choice ensures that one robot assembly will be better than the other in the worst case.

\section{Module Assembly Preference}

In modular robots, the choice of the joint locations on a link module will effect the kinematic capability of the entire assembly. It is useful to be able to specify the preference of certain link/joint assembly patterns in the overall assembly configuration in order to avoid undesirable kinematic conditions, such as link interference or joint redundancy. By joint redundancy, we mean that the number of effective DOF of a robot is less than the number of its joints. For example, assembly patterns with collinear revolute joint axes (Fig. 5) exhibit joint redundancy. However, the same assembly patterns may be emphasized in other situations. For

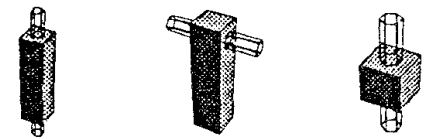

Figure 5: Joint patterns with redundancy

example, the redundant assembly patterns extend the dimension of the robot arm and enlarge the workspace. Besides, when one of the two joints fails, the other joint is able to drive that degree of freedom.

In order to retain or filter out a joint assembly patterns in a modular robot, a binary-valued function called the module assembly preference (MAP), $\phi$, is defined on a set of distinct assembly patterns. Let $\mathcal{F}$ represent the set of distinct assembly patterns. $[f] \in \mathcal{F}$ represents a distinct joint pattern.

Definition 3 A module assembly preference, $\phi: \mathcal{F} \rightarrow$ $\{0,1\}$, is a surjective function such that

$$
\phi:[f] \mapsto w, \quad w=0,1,
$$

$\phi([f])=0$ represents an undesirable joint pattern. The MAP can be thought of as a quantifiable rule varying with different kinematic requirements. The preference of an entire robot assembly configuration is defined based on a MAP of assembly patterns. Suppose the robot has $n$ links and the values of the MAP on Link $i$ is $w_{i}$. The structural preference of an entire robot, $\Phi$, is defined as follows.

Definition 4 The structural preference, $\Phi$, of a modular robot assembly configuration $A$ is the product of the MAPs of the robot's assembly patterns,

$$
\Phi(A)=\prod_{i=1}^{n} w_{i},
$$

where $A$ is the AIM of a robot assembly configuration. Since $w_{i}$ is equal to 0 or $1, \Phi(A)$ is equal to 0 or 1 as well. $\Phi(A)=0$ indicates the assembly configuration contains undesirable assembly patterns. The choice of a MAP, $\phi$, is illustrated in the following example.

Example 1 Let a 3-DOF fixed base serial type with $R$-joints and prismatic links satisfy the kinematic conditions: minimum link interference and no joint redundancy. The corresponding MAP is described in Fig. 6 . The intermediary prism link modules are connected by two joints. From [2] we obtain 9 distinct assembly patterns on link modules and 2 distinct patterns on cube modules. The choice of no joint redundancy implies that the MAP will be zero for patterns 4,9 , and 13 . The requirement on minimum link interference will set the MAP of patterns 2 and 7 to zero, since two joints are attached to the same end of the prism. The remaining patterns are assigned a MAP value of one. For the end prism link, there exists only two distinct assembly patterns (patterns 10 and 11). The requirements on the robot structure have little influence on the end link patterns, so their MAPs are set to 1 . If joint redundancy is allowed, patterns $4,9,13$ will be set to 1 . Using this MAP, the structural preferences of the 3 -DOF robot 


\begin{tabular}{|c|c|c|c|c|c|}
\hline ASSEMBLY PATTERNS & MAP & \multicolumn{2}{|l|}{ ASSEMBLY PATTERNS } & MAP \\
\hline 1 & 1 & 1 & 8 & & 1 \\
\hline 2 & & 0 & 9 & & 0 \\
\hline 3 & & 1 & 10 & & 1 \\
\hline 4 & & 0 & 11 & & 1 \\
\hline 5 & & 1 & 12 & & 1 \\
\hline 6 & 1 & 13 & & 0 \\
\hline 7 & & 0 & 14 & & 1 \\
\hline
\end{tabular}

Figure 6: MAP for patterns on a prism and a cube

configurations $A_{1}$ and $A_{2}$ can be obtained as follows.

$$
A_{1}=\left(\begin{array}{cccc}
1 & 0 & 0 & F B \\
1 & 2 & 0 & L \\
0 & 5 & 2 & L \\
0 & 0 & 2 & L \\
R & R & R & 0
\end{array}\right), A_{2}=\left(\begin{array}{cccc}
1 & 0 & 0 & F B \\
1 & 2 & 0 & L \\
0 & 1 & 9 & L \\
0 & 0 & 2 & L \\
R & R & R & 0
\end{array}\right)
$$

The structural preference of $A_{1}$ is calculated starting from the base module. Since there is only one assembly state on the fixed base (pattern 14), its MAP is set to 1. There are two R-joints connected to port 1 and 2 of link 2 (pattern 1 ), so $w_{2}=1$. R-joints are connected to port 2 and 5 of link 3 (pattern 5 ), so $w_{3}=1$. For the end link (pattern 10), $w_{4}=1$. Therefore, $\Phi\left(A_{1}\right)=$ $w_{2} \cdot w_{3} \cdot w_{4}=1$. Similarly, in $A_{2}, w_{2}=1$ (pattern 1), $w_{3}=0$ (pattern 8$)$, and $w_{4}=1$ (pattern 10$)$, so $\Phi\left(A_{2}\right)=0$.

\section{Assembly Configuration Eva. Function}

The structure of the ACEF for a serial modular robot is shown in Fig. 7. This function evaluates the "goodness" of a robot assembly configuration for a required task and structure specification. The "goodness" is represented by a non-negative real number. An AIM with large real value represents a good assembly configuration.

The input of the ACEF is an AIM with a predefined number of DOFs and predefined topology. The function is divided into task and structure parts. The MAP, $\phi$, is given to determine the structural preference $\Phi$. Task points and the TEC are given for task evaluation. In the first part of task evaluation, the workspace check procedure determines if an assembly, represented by an AIM, can carry out the specified task. If a task point is outside of the robot's workspace, there is no need to proceed with the task evaluation, and $\mu$ will be set to zero. If all task points are reachable, the TEC is applied to calculate $\mu$.

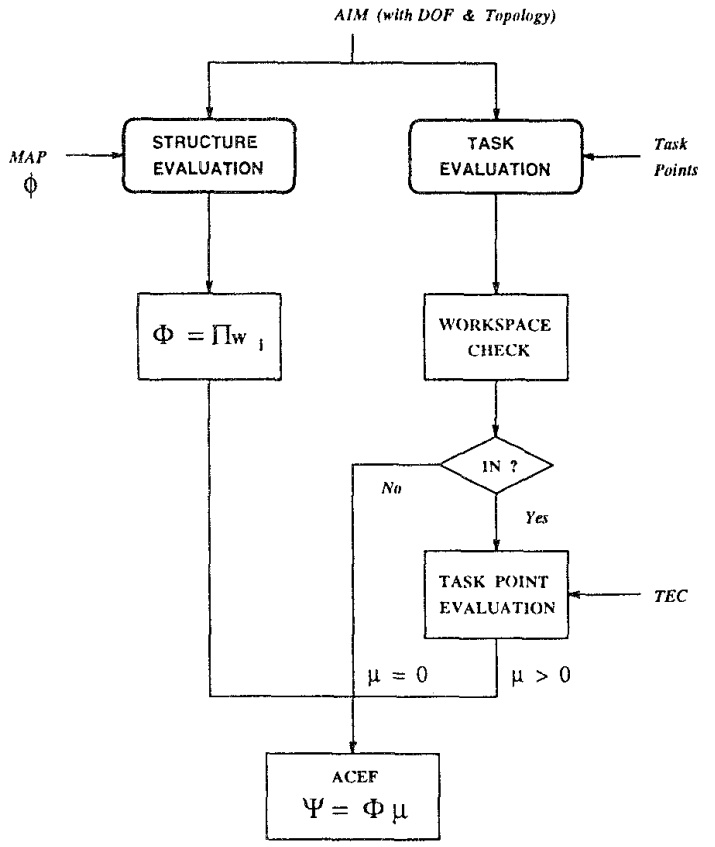

Figure 7: ACEF for serial modular robots

Definition 5 The ACEF, $\Psi$, for the performance of a robot assembly, $A$, is

$$
\Psi(A)=\Phi(A) \cdot \mu(A) .
$$

Since $\Phi(A)$ is equal to zero or one, and $\mu(A) \geq 0$, by definition, $\Psi(A) \geq 0$ as well. Note that the structure preference $\Phi$ functions as a filter for the ACEF. For assembly configurations satisfying the kinematic constraints, $\Phi$ is always equal to 1 . Therefore, $\Psi(A)=$ $\mu(A)$. Comparing the total performance of acceptable AIMs is equivalent to the comparison of the TECs of the task points only.

\section{WORKSPACE CHECK PROCEDURE}

The workspace check is accomplished by solving the inverse kinematics of the manipulator for a given task point. If a real valued solution can be found, the task point is within the workspace, otherwise, the task point is out of reach. A numerical inverse kinematics technique proposed by [7] is adopted here for its robustness and efficiency. The detail of the workspace checking can be found in [2].

\section{Genetic Algorithms for Modular Robots}

If the number of assembly configurations that can be generated from a given set of modules is small, an exhaustive evaluation of each assembly configuration can be performed in a reasonable amount of time. However, as the number of robot DOFs increases, the set of assembly configurations may become factorially large and the exhaustive search becomes undesirable. Instead, a discrete random search technique can be used for efficiency. Here, we explore the use of genetic algorithms (GA) to solve this problem. Other researchers 


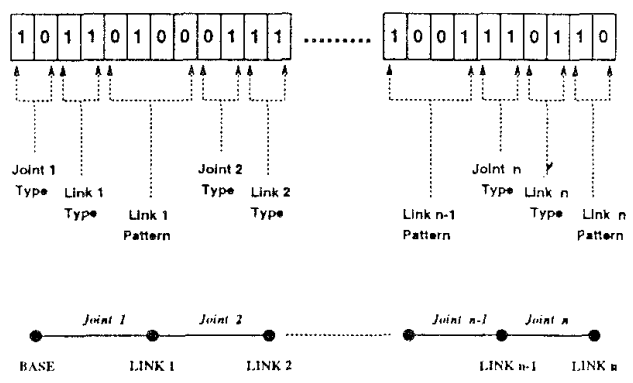

Figure 8: An assembly string representation

have applied GAs to distributed robotic systems and task-based robot design $[8,13]$.

A "genetic algorithm" is an optimization method based on a model of an ecological system in which the mechanics of natural selection and natural genetics are the primary factors for improving the performance of a population [6]. In this algorithm, candidate solutions are coded into string structures that are analogous to a genetic code. A fitness function will assign a fitness value to every string. The candidate strings are combined among themselves with a structured, yet randomized, information change in order to form a new generation of candidate solutions. The change is based on operations that mimic the adaptive process of natural systems: reproduction, crossover, and mutation. The strings of the new generation are created by using bits and pieces of the fittest strings in the previous generation. These bits and pieces of the string contribute to the overall performance of the string, and create offsprings with higher fitness values. It is interesting to note the analogy between the effect of module sub-assembly patterns on overall performance of a modular robot, and the influence of sub-strings on overall string fitness.

\section{CODING SCHEMES FOR AIMS}

To employ GAs effectively, a coding scheme to transform an AIM into a string structure is necessary. A binary string of $0 \mathrm{~s}$ or $1 \mathrm{~s}$ is chosen to represent an AIM because it can offer the maximum number of schemata per bit of any coding [6]. This string contains substrings, where each substring represents the types of joints and the types of link and joint patterns in an AIM. We call this binary string an assembly string. The lengths of the substrings are determined by the numbers of joint types and the number of distance joints and link patterns. Since joints and links are arranged in alternating sequence in a serial type robot, the substrings representing the types of joints and links are arranged in the same alternating sequence as in the AIM shown in Fig. 8.

Suppose the substring for the joint type is of length $l_{1}$. Each joint type is assigned a unique bit string pattern of length $l_{1}$. Similarly, a substring of length $l_{2}$ is used to represent the link type. The possible types of joint/link assembly patterns is assigned a substring of length $l_{3}$. In order to reduce the size of the search space, bit string patterns are only assigned to kinematically unique patterns. Note that in a serial robot, the number of joints connected to the intermediary links,

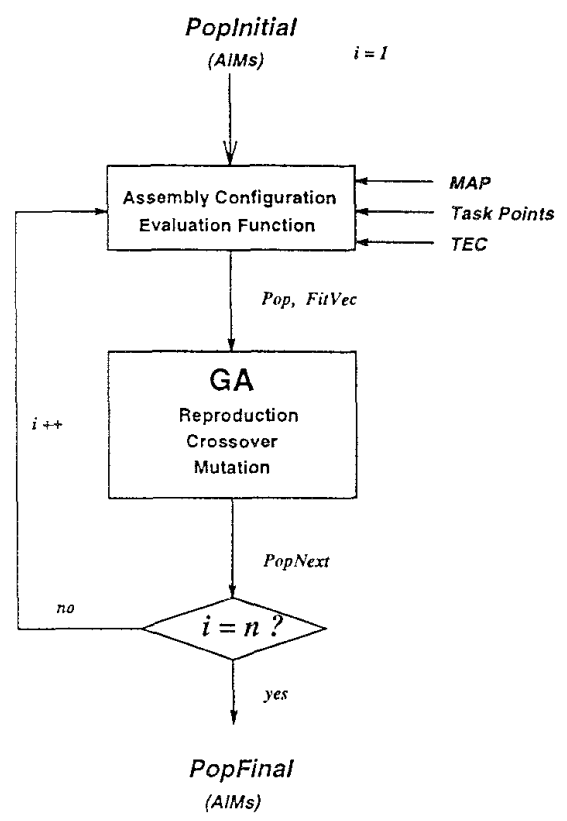

Figure 9: GA for task-optimal configurations

links $1, \cdots, n-1$, is different from the number of links attached to the end link, link $n$. Hence, the length of the joint pattern substring for link $n$ is different from that of links 1 to $n-1$. Let the length of this substring be $l_{4}$. Since there is only one way to attach a joint to the base of the robot, the base assembly pattern need not be specified in the assembly string. Hence, an $n$ DOF serial type robot can be expressed by an assembly string of length $n l_{1}+n l_{2}+(n-1) l_{3}+l_{4}$. If all joints (or all links) are of the same type, the joint (link) type substrings can be removed from the assembly string, i.e, $l_{1}=0$ (or $\left.l_{2}=0\right)$.

Because the number of distinct joint patterns is determined by the link module type, the joint pattern substring must be sufficiently long to represent the entire set of joint patterns. For some link module types, the number of distinct joint patterns which can be represented by this substring is much larger than the number of the actual distinct joint patterns. Hence, it is always possible to map an AIM into an assembly string, but the converse does not hold every time. For consistency, the fitness values of those assembly strings that cannot be mapped back to AIMs will be set to zero.

Fig. 9 depicts the application of GA in solving the taskoptimal configuration problem. The input is a set of randomly chosen assembly strings, PopInitial from the pool of kinematically unique assemblies provided by the algorithm of [1]. The number of elements in the set is specified by the user. The fitness function is the ACEF. The fitness value of every AIM in PopInitial. is obtained through an ACEF. A new generation of assembly strings is created by the GA operations (refer to [6] for detail), and are then presented to the ACEF for fitness evaluation. The whole process will repeat until a predetermined generation, i.e., PopFinal in the 


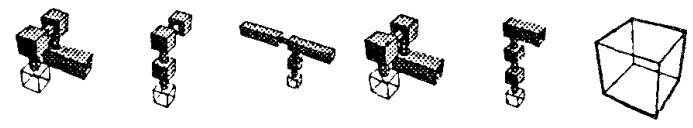

Figure 10: Initial configurátions

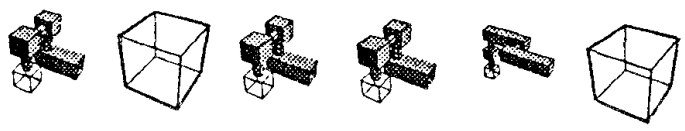

Figure 11: Final configurations

figure, is reached. After the destination generation is reached, we choose the assembly string in this generation that has the largest fitness value as the optimal assembly configuration satisfying the required task and structure specifications.

Example 2 We wish to find a 3-DOF fixed base serial robot with R-joints that passes through a set of task points listed in the following table. We also demand that there are no redundant joints and minimum link interference. The TEC is chosen to be the manipulator's manipulability measure and the MAP is described in Example 1.

\begin{tabular}{|c|c|c|c|}
\hline \multicolumn{2}{|c|}{ Task Point } & \multicolumn{2}{c|}{ Task Point } \\
\hline 1 & $(3.0,0.5,0.5)$ & 4 & $(2.0,2.0,0.5)$ \\
\hline 2 & $(3.0,0.5,1.5)$ & 5 & $(0.5,3.0,0.5)$ \\
\hline 3 & $(2.0,2.0,1.5)$ & 6 & $(0.5,3.0,1.5)$ \\
\hline
\end{tabular}

The initial set of AIMs is shown in Fig. 10. The empty boxes represent assembly strings that do not correspond to any AIM. Their fitness values are set to zero. The fitness values of these AIMs obtained from the ACEF are $(5.7963,0 ., 0 ., 5.7963,0 ., 0$.). The parameters used in the GA are $P_{\text {cross }}=0.6$, the probability of crossover operation, and $P_{\text {mutate }}=0.1$, the probability of mutation. The destination generation is chosen to be the $10^{t h}$ generation. After evolving ten generations, the AIMs in the final generation are shown in Fig. 11. Their fitness values are $(5.7963,0 ., 5.7963,5.7963,0 ., 0$.$) . Fig. 12$ shows the average and maximum fitness value in every generation. Assembly configurations 1,3 , and 4 are identical and have the highest fitness value of 5.7963 so they are chosen as the optimal one.

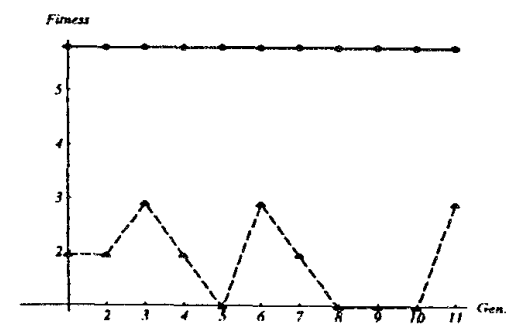

Figure 12: Avg. \& max. fitness in each generation

\section{Conclusion}

In order to evaluate a modular robot assembly configuration, we introduced a flexible and general assembly configuration evaluation function. This function can be used in two ways to find the optimal modular robot assembly configuration that solves a given task. First, the function could be used to evaluate the suitability of each of the kinematically unique assembly configuration that is enumerated by the algorithm in [1]. The configuration with the highest evaluation is obviously the most suitable. However, in some instances, such a procedure will be computationally expensive. Instead, a discrete combinatorial optimization algorithm, which employs the modular robot evaluation function, is a desirable alternative. We have found the Genetic Algorithm method to be especially well suited for the modular robot problem, and we presented a method to effectively encode modular robot assembly configurations in the bit strings required for the GA procedure.

\section{References}

[1] I.-M. Chen and J. W. Burdick, "Enumerating NonIsomorphic Assembly Configurations of Modular Robotic Systems," Proc. of IROS, Yokohama, Japan, pp. 1985$1992,1993$.

[2] 1.-M. Chen, "Theory and Applications of Modular Reconfigurable Robotic Systems," PhD Dissertation, California Institute of Technology, 1994.

[3] R. Cohen, M. G. Lipton, M. Q. Dai and B. Benhabib, "Conceptual Design of a Modular Robot," ASME J. Mechanical Design, 114, pp. 117-125,March, 1992.

[4] L. Dobrjanskyj and F. Freudenstein, "Some Applications of Graph Theory to the Structural Analysis of Mechanisms," ASME J. Engineering for Industry, 89, pp 153158, Feb., 1967.

[5] T. Fukuda and S. Nakagawa, "Dynamically Reconfigurable Robotic System," Proc. IEEE Int. Conf. Robotics and Automation, pp 1581-1586, 1988.

[6] D. E. Goldberg, "Genetic Algorithms in Search, Optimization, and Machine Learning," Addison-Wesley, 1989.

[7] P. K. Khosla, C. P. Neuman and F. B. Prinz, "An Algorithm for Seam Tracking Applications," Int. J. Robotics Research, 4, No. 1, pp 27-41, 1985.

[8] J. O. Kim and P. Khosla, "Design of Space Shuttle Tile Servicing Robot: An Application of Task Based Kinematic Design," Proc. IEEE Int. Conf. Robotics and Automation, pp 867-874, 1993.

[9] C. A. Klein and B. E. Blaho, "Dexterity Measures for the Design and Control of Kinematically Redundant Manipulators," Int J Robotics Res., 6, No. 2, pp 72-83, 1987. [10] C. A. Klein and 'T. A. Miklos, "Spatial Robotic Isotropy," Int J Robotics Res., 10, No.4, pp 426-437, 1991. [11] C. Paredis and P. Khosla, "Kinematic Design of Serial Link Manipulators From Task Specifications," Int. J. Robotics Research, 12, No.3, pp 274-287, 1993.

[12] D. Schmitz, P. Khosla and T. Kanade, "The CMU Reconfigurable Modular Manipulator System," Carnegie Mellon Univ., CMU-RI-TR-88-7, 1988.

[13] T. Ueyama, 'T. Fukuda and F. Arai, "Structure Configuration Using Genetic Algorithm for Cellular Robotic System," Proc. of IROS, Raleigh, NC, pp 1542-1549, 1992.

[14] K. H. Wurst, "The Conception and Construction of a Modular Robot System," Proc. 16th Int. Sym. Industrial Robotics (ISIR), pp 37-44, 1986.

[15] T. Yoshikawa, "Manipulability of Robotic Mechanisms," Int. J. Robotics Research, 4, No.2, pp3-9, 1985.

[16] N. Deo, Graph Theory with Applications to Engineering and Computer Science, Prentice-Hall, 1974. 